\title{
What does (not) drive the variation of the low-mass end of the stellar initial mass function of early-type galaxies
}

\author{
C. E. Barbosa ${ }^{1}$, C. Spiniello ${ }^{2,3}$, M. Arnaboldi ${ }^{4}$, L. Coccato ${ }^{4}$, M. Hilker ${ }^{4}$, and T. Richtler ${ }^{5}$ \\ 1 Universidade de São Paulo, IAG, Departamento de Astronomia, Rua do Matão 1226, São Paulo, SP, Brazil \\ e-mail: carlos.barbosa@usp.br \\ 2 Department of Physics, University of Oxford, Denys Wilkinson Building, Keble Road, Oxford OX1 3RH, UK \\ 3 INAF, Osservatorio Astronomico di Capodimonte, Via Moiariello 16, 80131 Naples, Italy \\ 4 European Southern Observatory, Karl-Schwarzschild-Straße 2, 85748 Garching, Germany \\ 5 Departamento de Astronomia, Universidad de Concepción, Concepción, Chile
}

Received 30 October 2020 / Accepted 1 December 2020

\begin{abstract}
Context. The stellar initial mass function (IMF) seems to be variable and not universal, contrary to what has been argued in the literature over the last three decades. Several relations of the low-mass end of the IMF slope with other stellar population, photometrical, and kinematical parameters of massive early-type galaxies (ETGs) have been proposed, but consensus on the factual cause of the observed variations has not yet been reached.

Aims. We investigate the relationship between the IMF and other stellar population parameters in NGC 3311, the central galaxy of the Hydra I cluster. NGC 3311 is a unique laboratory, characterized by old and metal-rich stars, that is similar to other massive ETGs for which the IMF slope has been measured to be bottom-heavy (i.e., dwarf-rich); however, it has unusual stellar velocity dispersion and $[\alpha / \mathrm{Fe}]$ profiles, both of which increase with radius.

Methods. We use the spatially resolved stellar population parameters (age, total metallicity, and $[\alpha / \mathrm{Fe}]$ ) that were derived in a forthcoming paper (Barbosa et al. 2020) - via the full-spectrum fitting of high signal-to-noise MUSE observations - to compare the IMF slope in the central part of NGC $3311(R \lesssim 16 \mathrm{kpc})$ against other stellar parameters, with the goal of assessing their relations and dependencies.

Results. For NGC 3311, we unambiguously invalidate the previously observed direct correlation between the IMF slope and the local stellar velocity dispersion, confirming some doubts that had been raised in the literature. This relation may simply arise as a spatial coincidence between the region with the largest stellar velocity dispersion and the region where the oldest in situ population is found and dominates the light. We also show robust evidence that the proposed IMF-metallicity relation is contaminated by the degeneracy between these two parameters. We do confirm that the stellar content in the innermost region of NGC 3311 follows a bottom-heavy IMF, in line with other literature results. The tightest correlations we found are those between stellar age and the IMF and between the galactocentric radius and the IMF.

Conclusions. The variation of the IMF at its low-mass end is not due to kinematical, dynamical, or global properties in NGC 3311. We speculate instead that the IMF might be dwarf-dominated in the "red nuggets" that formed through a very short and intense star formation episode at high redshifts $(z>2)$ when the Universe was denser and richer in gas, and which then ended up being the central cores of today's giant ellipticals.
\end{abstract}

Key words. galaxies: clusters: individual: Hydra I - galaxies: individual: NGC 3311 - galaxies: elliptical and lenticular, cD galaxies: kinematics and dynamics - galaxies: structure - galaxies: stellar content

\section{Introduction}

The initial mass function (IMF) is the distribution of stars per unit of mass formed in a volume of space within a single starformation event. Since the mass of a star determines its subsequent evolutionary path, the IMF influences all the observable properties of a stellar system. In addition, the IMF slope at the low-mass end modifies the stellar mass-to-light ratio value in any galaxy. In fact, for galaxies dominated by an old stellar population, low-mass stars account for more than half of the mass budget but contribute very little to the integrated luminosity (see, e.g., Fig. 2 in Conroy \& van Dokkum 2012a). This makes the characterization of the low-mass IMF slope from integrated light a challenging task, though one that is crucial for assessing the relative contribution of stars and dark matter as well as for understanding their relative distribution in the baryon-dominated inner galaxy regions.

Over the last decade, a large number of studies have reported that the low-mass end of the IMF slope in massive early-type galaxies (ETGs) is bottom-heavier (i.e., more dwarf-rich) than the IMF slope inferred in the Milky Way (MW) via a variety of methods, such as stellar populations, gravitational lensing, and dynamics (see Cenarro et al. 2003; van Dokkum \& Conroy 2010; Treu et al. 2010; Conroy \& van Dokkum 2012b; Spiniello et al. 2012, 2014; Cappellari et al. 2012; La Barbera et al. 2013, 2017). However, a general consensus regarding which physical quantity is really responsible for the non-universality of the IMF is far from being reached. The stellar velocity dispersion 
(Cappellari et al. 2012; Spiniello et al. 2014), the total mass density (Spiniello et al. 2015), the metallicity (Martín-Navarro et al. 2015a), the magnesium abundance (La Barbera et al. 2017), the age, and the $\alpha$-element abundance (McDermid et al. 2014) have been investigated so far.

More recently, spatially resolved studies have found that the bottom-heavy IMF is concentrated in the innermost region of galaxies (Martín-Navarro et al. 2015b; van Dokkum et al. 2017; Sarzi et al. 2018; Parikh et al. 2018; Vaughan et al. 2018; La Barbera et al. 2019), indicating that local properties, not just global properties, may drive variation at the low-mass end of the IMF. These findings are particularly interesting in the context of the two-phase formation scenario (Naab et al. 2009; Oser et al. 2010; Hilz et al. 2012; Rodriguez-Gomez et al. 2016; Pulsoni et al. 2020) since the center is where the in situ population, formed during the first phase of the mass assembly, dominates the light.

The majority of massive ETGs show mostly flat or falling stellar velocity dispersion $\left(\sigma_{*}\right)$ profiles (Fisher et al. 1995; Sembach \& Tonry 1996; Carter et al. 1999; Brough et al. 2007; Emsellem et al. 2014). Consequently, the IMF has always been measured to be bottom-heavy in the region where the $\sigma_{*}$ profile had its central peak. The same holds for the $[\alpha / \mathrm{Fe}]$ abundance, which is often larger in the center than in the outer regions. Thus it had not been possible until now to independently assess whether the spatial variation of the low-mass end of the IMF is driven by the kinematics (i.e., the IMF is dwarf-rich wherever $\sigma_{\star}$ is high), the stellar populations (i.e., the IMF is dwarf-rich when the metallicity or $[\alpha / \mathrm{Fe}]$ is super solar), or by other mechanisms linked to the intense early star formation (i.e., the IMF is dwarf-rich in the innermost region where the in situ stars dominate, despite the local kinematics).

In this context, NGC 3311, the central galaxy of the Hydra I cluster, is a perfect "laboratory" for disentangling between these scenarios, having both a $\sigma_{\star}$ and a $[\alpha / \mathrm{Fe}]$ profile that rise with increasing galactocentric distances ${ }^{1}$, but a total metallicity profile that decreases instead (Barbosa et al. 2016, hereafter B16). The very peculiar profiles in NGC 3311 are the results of the presence of multiple components with distinct kinematics and stellar populations, as we show in detail in a forthcoming paper (Barbosa et al. 2020, hereafter B20). There, we performed a fully Bayesian, full-spectral fitting analysis on high signal-to-noise MUSE spectra and obtained accurate spatially resolved measurements of light-weighted age, metallicity $([\mathrm{Z} / \mathrm{H}]), \alpha$-element abundance $([\alpha / \mathrm{Fe}])$, and IMF slope $\left(\Gamma_{\mathrm{b}}\right)$. More details regarding the stellar population modeling are available in B20. Here, we focus mainly on the low-mass end of the IMF slope and its possible correlations with the other kinematical and stellar population parameters. We note that the spatially resolved stellar population properties of NGC 3311 cover a range of metallicities, $[\alpha / \mathrm{Fe}]$ abundances, and $\sigma_{\star}$ that would allow us to observe IMF gradients and correlations if the IMF does indeed depend on these parameters, as has been done in previous studies.

\section{What drives the IMF variations}

In B20, we used the E-MILES (Vazdekis et al. 2016) stellar population models with a "bimodal" IMF (Vazdekis et al. 1996), which is obtained by changing the high-mass end $\left(M \gtrsim 0.6 M_{\odot}\right)$ power-law slope $\left(\Gamma_{\mathrm{b}}\right)$. The IMF is then normalized to $1 M_{\odot}$ since

\footnotetext{
1 We note that few other massive galaxies with rising $\sigma_{*}$ profiles exist (see e.g., Veale et al. 2017).
}

more massive stars are already dead in old stellar populations; therefore, a change in the $\Gamma_{\mathrm{b}}$ slope can be interpreted as a change in the ratio between stars with masses above and below $0.6 M_{\odot}$. In practice, we are constraining the "present-day" mass function between 0.1 and 1 solar masses.

In the next sections, we try to understand what the possible physical causes for the non-universality of the IMF in NGC 3311, as well as in other massive ETGs, may be. To this purpose, we compare the results from NGC 3311 (obtained in B20, shown in all figures as data points color-coded by distance from the center) with those obtained globally across different galaxies (dashed lines in all plots) and spatially within one or more objects (solid lines in all plots).

\subsection{Correlations that do not hold for NGC 3311}

The stellar velocity dispersion was one of the first "drivers" proposed to explain the bottom-heaviness of the IMF both across different galaxies (van Dokkum \& Conroy 2010; Treu et al. 2010; Conroy \& van Dokkum 2012b; Cappellari et al. 2012; Spiniello et al. 2014) and within individual objects (MartínNavarro et al. 2015b; Sarzi et al. 2018; La Barbera et al. 2019). However, recent indications have called into question the $\mathrm{IMF}-\sigma_{\star}$ relation, mainly due to the fact that the spatial gradients of the two quantities were often very different in radial extension (e.g., Sarzi et al. 2018). But, above all, the IMF slope has never been measured in galaxies like NGC 3311 that have rising $\sigma_{*}$ and $[\alpha / \mathrm{Fe}]$ profiles.

In the upper panel of Fig. 1, we decidedly show that a direct linear correlation between the IMF bottom-heaviness and $\sigma_{\star}$ does not hold for NGC 3311. In this panel, we compare the results from B20 with those obtained for M 87 with MUSE (red line, Sarzi et al. 2018), and with a sample of galaxies stacked in three mass bins (green lines, Parikh et al. 2018) observed by the Mapping Nearby Galaxies at Apache Point Observatory (MaNGA) survey (Bundy et al. 2015). We also show the global results obtained in La Barbera et al. (2013, pink dashed line), which were based on a spectroscopic study of a large sample ( 24 000) of ETGs from the Spheroids Panchromatic Investigation in Different Environmental Regions (SPIDER) survey (La Barbera et al. 2010), which used the same stellar population models (and the same IMF) as used in B20. While a clear direct correlation is visible for all previous studies, a mild anticorrelation is inferred for NGC 3311, with the IMF slope being more bottom-heavy for points with lower $\sigma_{\star}$. However, the IMF is dwarf-rich in the center of NGC 3311. As we will further show in the next section, all the results support a conjecture where the innermost region of massive galaxies is characterized by a dwarf-rich IMF regardless of their kinematical properties.

Another parameter that has been reported to correlate directly with the IMF slope is the total metallicity $([\mathrm{Z} / \mathrm{H}]$, Martín-Navarro et al. 2015a). Tentatively, a correlation can be seen for NGC 3311 in the lower panel of Fig. 1, which is in agreement with the global results from the Calar Alto Legacy Integral Field Area (CALIFA, Sánchez et al. 2012) collaboration by Martín-Navarro et al. (2015a) as well as with the spatially resolved results obtained for M 87 and the stacked MaNGA galaxies. These observational results suggest that the IMF is bottom-heavy for a more metal-rich population. Although we reproduce this relation, we also show that the slope of the correlation is almost perfectly aligned with the mean posterior distribution between the two parameters obtained in B20, which is shown as an dashed ellipsoidal line in the figure. Therefore, there are strong grounds to believe that the relation is 

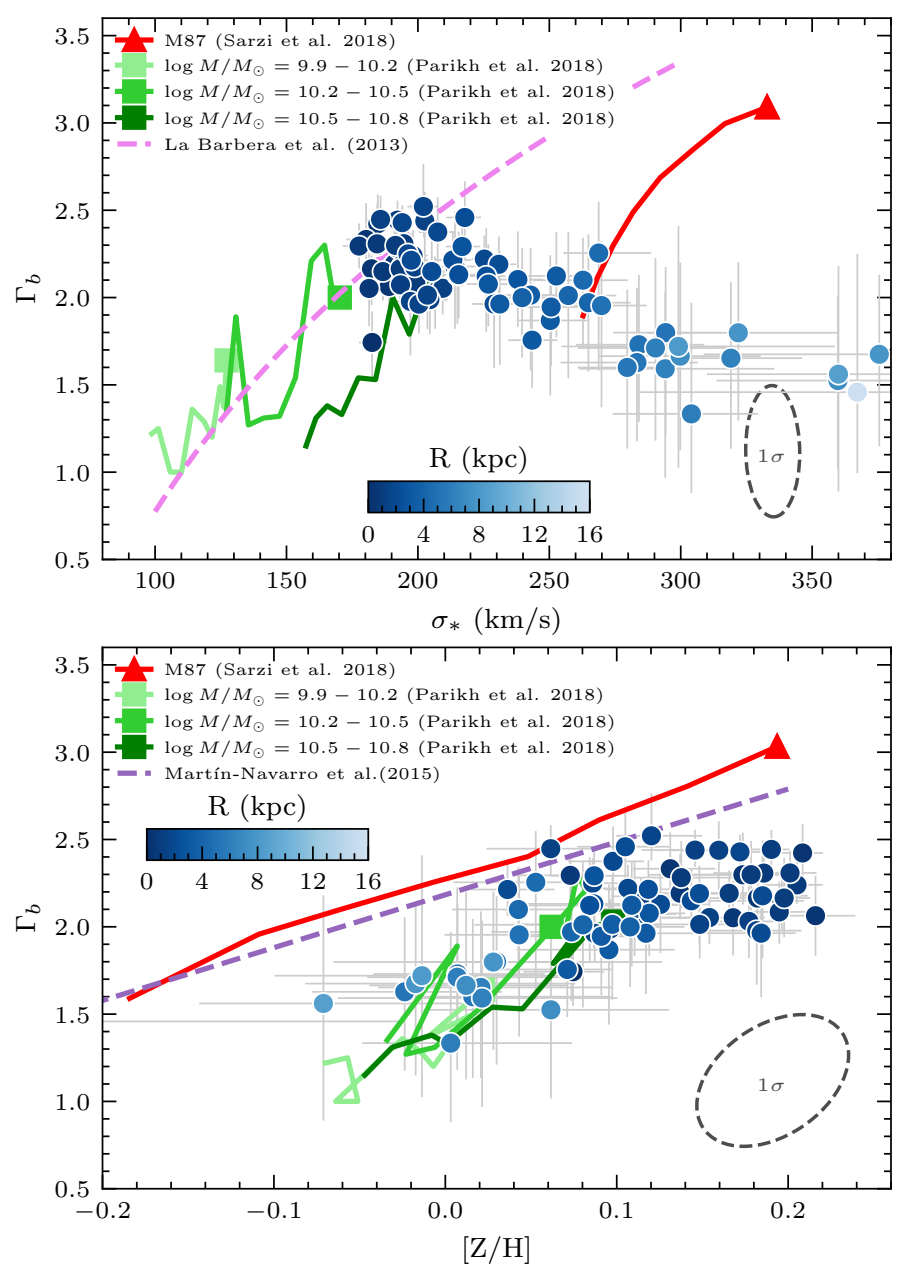

Fig. 1. IMF slope $\left(\Gamma_{\mathrm{b}}\right)$ versus stellar velocity dispersion $\left(\sigma_{\star}\right.$, upper panel) and total metallicity $([\mathrm{Z} / \mathrm{H}]$, bottom panel). Blue circles indicate data points for NGC 3311, colored according to the galactocentric distance. For other works, solid lines refer to local variations within galaxies (with a symbol indicating the center of the objects) and dashed lines refer to global variation across galaxies.

at least partially due to an observational degeneracy between the two plotted quantities ${ }^{2}$. Furthermore, from a theoretical perspective, simulations have demonstrated that the trends predicted by the IMF- $[\mathrm{Z} / \mathrm{H}]$ correlation fail to reproduce the general trend observed between the iron and $\alpha$-element abundances (Gutcke \& Springel 2019). Finally, we also note that other recent observational results (e.g., Martín-Navarro et al. 2019) indicate that the metallicity does not trace the IMF in spatially resolved observations $^{3}$.

\subsection{Correlations that do hold for NGC 3311}

In Fig. 2, we plot the observed relations between the IMF slope and the radius (normalized by the effective radius $R_{\mathrm{e}}$, top panel), stellar age (middle panel), and surface stellar density (bottom

\footnotetext{
2 This evidence reinforces the importance of committing to a fully Bayesian approach and carefully inspecting the posterior probability results, not simply as a way to determine point estimates and uncertainties but also as a tool for understanding our limitations from an observational perspective.

3 The results in Martín-Navarro et al. (2019) suggest that the metallicity tracks the distribution of cold orbits, at least for the galaxy FCC 167, whereas the IMF slope tracks the distribution of warm orbits.
}

panel). As in the previous figures, we compare the data points that show the NGC 3311 results (obtained in B20) with lines showing the relations reported in the literature. Interestingly, these three plots support the following statement: despite the different kinematical properties of the galaxies' central regions, the IMF is always dwarf-rich within the innermost few kiloparsecs. In these regions, according to the so-called two-phase formation scenario, the old and very dense "red nugget" that formed during the first phase of the mass assembly dominates the light.

We therefore argue, in line with what has been suggested by other authors (van Dokkum et al. 2017; Martín-Navarro et al. 2019; Smith 2020), that the excess of dwarf stars may have originated from the first phase of galaxy formation in the early Universe, which was characterized by high density, temperatures, and gas turbulence. These are, according to theoretical works (Hopkins 2012; Chabrier et al. 2014), key parameters that drive the fragmentation of molecular clouds (higher density and temperature should make the fragmentation easier, leading to the formation of more dwarf stars, i.e., a bottom-heavier $\mathrm{IMF})^{4}$. Later accretions of less massive satellites with an "MWlike" IMF (because they formed later on, under less extreme temperature and density conditions) would settle preferentially in the galaxy periphery due to the lower densities and masses of the accreted systems and thus create a spatial IMF gradient (Spavone et al. 2017; Pulsoni et al. 2020) ${ }^{5}$. Finally, our findings are consistent with those reported in Conroy \& van Dokkum (2012b) and La Barbera et al. (2019), who found that the IMF becomes increasingly bottom-heavy either when the star formation timescale becomes shorter (see Sect. 2.3), or when the surface density and/or the interstellar medium pressure increase.

\subsection{The (apparently) puzzling case of the $[\alpha / \mathrm{Fe}]$ abundance}

According to the scenario described above, and in line with the results of B20, the central core of NGC 3311 is a red nugget that formed its stars under extreme conditions. Such a short star formation timescale would lead to a high $[\alpha / \mathrm{Fe}]$ abundance (Thomas et al. 2005). This is explained by the fact that the star formation quenching took place before the Type Ia supernova explosions were able to pollute the interstellar medium with iron.

The $\alpha$-abundance of stars in the innermost region of NGC 3311 is not extremely high $(0.08 \pm 0.02, \mathrm{~B} 20)$. In principle, this might raise doubts about our conclusions that the stars in the core of NGC 3311 were formed via a burst during the first phase of the two-phase scenario and with a dwarf-rich IMF. However, as shown in Fig. 8 of B20, the NCG 3311 core requires a very large $[\mathrm{Mg} / \mathrm{Fe}](>0.3)$, which is, in practice, what was measured in Thomas et al. (2005) since they derived $[\alpha / \mathrm{Fe}]$ from the absorption line indices $\mathrm{Mg} b$ and $\langle\mathrm{Fe}\rangle$. The same is valid for the measurements performed by McDermid et al. (2014), who also proposed a direct global correlation between the IMF slope and the $[\alpha / \mathrm{Fe}]$ but actually measured $[\mathrm{Mg} / \mathrm{Fe}]$ instead $^{6}$. Hence, once again, the direct correlation found between the IMF slope and

4 A further parameter has very recently been advocated by Davis \& van de Voort (2020): the abundance of deuterium in the birth clouds of forming stars.

5 In NGC 3311, the later mass accretion scenario is supported by the rising velocity dispersion profile, which has been considered the "smoking gun" of recent accretion of low-mass systems, dominating the dispersion scatter and leading to larger $\sigma_{\star}$ at larger distances (Hilker et al. 2018).

6 We refer the reader to B20 for more details about the different weight one gives to the $\alpha$-elements when using line indices or when using full spectral fitting. 

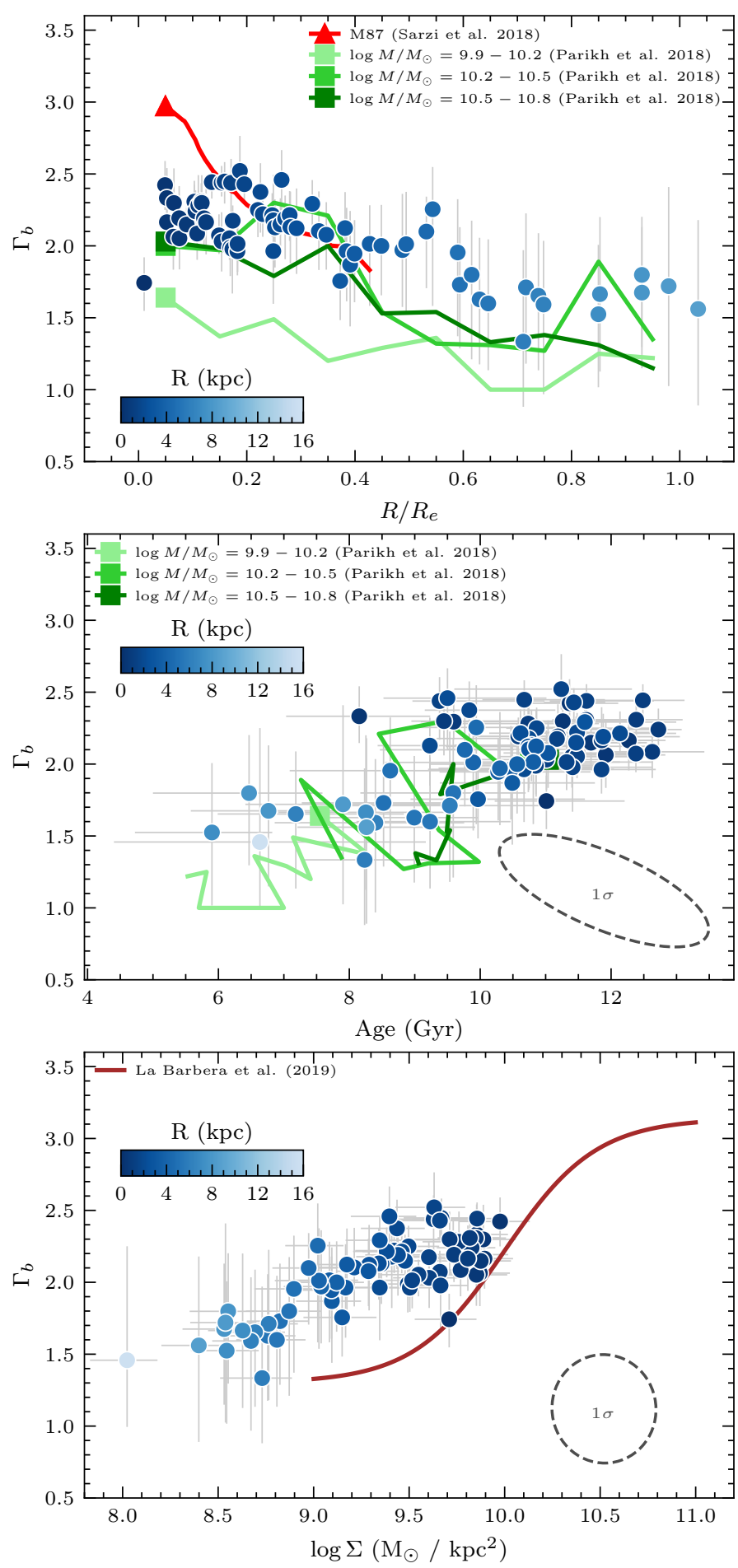

Fig. 2. IMF spatial gradient (upper panel), the correlation between $\Gamma_{\mathrm{b}}$ and the stellar age (middle panel), and the correlation between $\Gamma_{\mathrm{b}}$ and the total stellar density (bottom panel). All panels suggest that the IMF slope is only bottom-heavy for the in situ stars, which formed at very high redshift in the first phase of the two-phase formation scenario.

the $[\alpha / \mathrm{Fe}]$ (or, more exactly, $[\mathrm{Mg} / \mathrm{Fe}]$ ) in other ETGs, including $\mathrm{M} 87$, is found; this is because, for the majority of the massive ETGs, the region where the $[\mathrm{Mg} / \mathrm{Fe}]$ is at its maximum spatially coincides with the region where the in situ pristine population of stars dominates the light. We can now prove such a statement thanks to the unusual mass assembly of NGC 3311 (see B20 for more details), which caused an inverse $\alpha$-element pro-

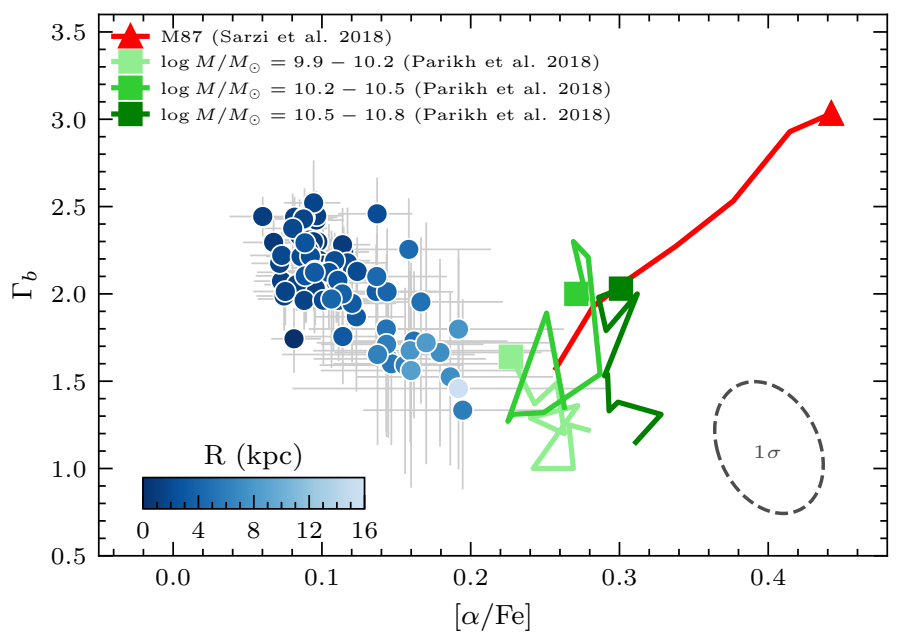

Fig. 3. MF slope $-[\alpha / \mathrm{Fe}]$ relation. For NGC 3311 (blue points), these two quantities are anti-correlated. This is the opposite of what happens for M 87 (red). However, we stress that in B20 we used full-spectral fitting, rather than line indices as in Sarzi et al. (2018) for M 87, thus measuring a different quantity (using indices, one is much more sensitive to $\mathrm{Mg}$ than to the "bulk" changes in all $\alpha$-elements). We note that the $[\alpha / \mathrm{Fe}]$ estimates obtained in Barbosa et al. (2018) from the same data but using line indices imply a high value, which would shift the blue data points toward the red and green lines. The situation is much more complicated for the results based on MaNGA (green lines), where different galaxies, with possibly different $[\alpha / \mathrm{Fe}]$ profiles, are stacked together.

file. In fact, in our case, $\Gamma_{\mathrm{b}}$ decreases when $[\alpha / \mathrm{Fe}]$ increases, as shown in Fig. 3. We stress that the offset between our results and those of Sarzi et al. (2018) and Parikh et al. (2018) is due to the fact that we measure variations in all the $\alpha$-elements, rather than only $\mathrm{Mg}$. The $[\mathrm{Mg} / \mathrm{Fe}]$ in the innermost region of NGC 3311 is indeed large (see Barbosa et al. 2018 and B20), thus these stars are compatible with having been formed via a burst of star formation. Finally, we note that the large $[\alpha / \mathrm{Fe}]$ measured in the galactic halo of NGC 3311 is not caused by the fact that these stars formed at high- $z$ in situ via a burst: it is instead caused by the late accretion of previously quenched satellites (see B20 for more details)

In conclusion, all evidence consistently points to the fact that the IMF is dwarf-rich when stars are formed through an intense and brief high- $z$ star formation episode.

\section{Summary and conclusion}

In this Letter, we used spatially resolved kinematical and stellar population results from NGC 3311, the central galaxy of the Hydra I cluster, to address the question of whether the relations between the low-mass end of the IMF slope and kinematical $\left(\sigma_{\star}\right)$ or stellar population (age, $[\mathrm{Z} / \mathrm{H}]$, and $\left.[\alpha / \mathrm{Fe}]\right)$ parameters are real, physically motivated relations. We find that the IMF- $\sigma_{\star}$ relation is mainly a spatial coincidence arising from the fact that the majority of massive ETGs show a $\sigma_{\star}$ profile that is peaked in the center, likely because of violent relaxation (Hilz et al. 2012). This is not true for NGC 3311, but its core IMF is nonetheless bottom-heavy. The strength of the direct IMF-metallicity correlation may instead be augmented by the degeneracy between $\Gamma_{b}$ and $[\mathrm{Z} / \mathrm{H}]$, as addressed more extensively in the companion paper B20.

We also presented our arguments in support of a two-phase formation scenario with two IMFs with different low-mass end 
slopes for the two distinct phases, as suggested by Smith (2020). This scenario is able to reconcile all the different observational and theoretical evidence in a spatially resolved sense within a single galaxy, as well as in a "global" sense on large galaxy samples. Studying the IMF spatial gradients, the relation that links its slope $\Gamma_{\mathrm{b}}$ to the stellar age, and the relation between $\Gamma_{\mathrm{b}}$ and stellar surface density, we explain the bottom-heaviness of the IMF as a characteristic of the "relic" nucleus that was formed during the first phase at very high- $z$ in a short, violent star formation episode $\left(\tau \sim 100 \mathrm{Myr}, \mathrm{SFR} \geq 10^{3} M_{\odot} \mathrm{yr}^{-1}\right)$. The accreted stellar component, which dominates the light budget at larger galactocentric radii and was formed under less extreme and more time-extended star formation episodes (second phase), is instead described by a standard MW-like IMF.

This version of the two-phase formation scenario naturally explains the radial IMF gradients recently reported for "normal" massive ETGs, and the very recent results obtained for relic galaxies provide further independent support for this idea. Relics are galaxies that are rare, local, ultra-compact, massive, red, and dead and which missed the second phase of accretion, thus evolving passively and unperturbed after the first burst of star formation. Since they missed this second phase, relics should have a bottom-heavy IMF everywhere; this is exactly what was reported by Martín-Navarro et al. (2015c) and FerréMateu et al. (2017) for the only three relic systems for which spatially resolved stellar population analysis has been performed to date. The outlook is bright, thanks to the newly initiated INSPIRE Project (Spiniello et al. 2021), which aims to confirm a larger number of relics at $z<0.5$ and precisely infer their IMF slopes thanks to high signal-to-noise ratio X-shooter spectra.

Acknowledgements. The authors wish to acknowledge the anonymous referee for the constructive report. The authors acknowledge C. Mendes de Oliveira for discussions and suggestions. CEB gratefully acknowledges the São Paulo Research Foundation (FAPESP), grants 2011/51680-6, 2016/123310, 2018/24389-8. CS is supported by a Hintze Fellowship at the Oxford Centre for Astrophysical Surveys, which is funded through generous support from the Hintze Family Charitable Foundation. TR acknowledges support from the BASAL Centro de Astrofisica y Tecnologias Afines (CATA) PFB-06/2007. This work is based on observations collected at the European Organisation for Astronomical Research in the Southern Hemisphere under ESO programme 094.B-0711(A). It has made use of the computing facilities of the Laboratory of Astroinformatics (Instituto de Astronomia, Geofísica e Ciências Atmosféricas, Departamento de Astronomia/USP, NAT/Unicsul), whose purchase was made possible by FAPESP (grant 2009/54006-4) and the INCT-A. This research has made use of the NASA/IPAC Extragalactic Database (NED), which is operated by the Jet Propulsion Laboratory, California Institute of Technology, under contract with the National Aeronautics and Space Administration.

\section{References}

Barbosa, C. E., Arnaboldi, M., Coccato, L., et al. 2016, A\&A, 589, A139 Barbosa, C. E., Arnaboldi, M., Coccato, L., et al. 2018, A\&A, 609, A78 Barbosa, C. E., Spiniello, C., Arnaboldi, M., et al. 2020, A\&A, submitted Brough, S., Proctor, R., Forbes, D. A., et al. 2007, MNRAS, 378, 1507
Bundy, K., Bershady, M. A., Law, D. R., et al. 2015, ApJ, 798, 7

Cappellari, M., McDermid, R. M., Alatalo, K., et al. 2012, Nature, 484, 485

Carter, D., Bridges, T. J., \& Hau, G. K. T. 1999, MNRAS, 307, 131

Cenarro, A. J., Gorgas, J., Vazdekis, A., Cardiel, N., \& Peletier, R. F. 2003, MNRAS, 339, L12

Chabrier, G., Hennebelle, P., \& Charlot, S. 2014, ApJ, 796, 75

Conroy, C., \& van Dokkum, P. 2012a, ApJ, 747, 69

Conroy, C., \& van Dokkum, P. G. 2012b, ApJ, 760, 71

Davis, T. A., \& van de Voort, F. 2020, MNRAS, 498, 4051

Emsellem, E., Krajnovic, D., \& Sarzi, M. 2014, MNRAS, 445, L79

Ferré-Mateu, A., Trujillo, I., Martín-Navarro, I., et al. 2017, MNRAS, 467, 1929

Fisher, D., Illingworth, G., \& Franx, M. 1995, ApJ, 438, 539

Gutcke, T. A., \& Springel, V. 2019, MNRAS, 482, 118

Hilker, M., Richtler, T., Barbosa, C. E., et al. 2018, A\&A, 619, A70

Hilz, M., Naab, T., Ostriker, J. P., et al. 2012, MNRAS, 425, 3119

Hopkins, P. F. 2012, MNRAS, 423, 2037

La Barbera, F., de Carvalho, R. R., de La Rosa, I. G., et al. 2010, MNRAS, 408, 1313

La Barbera, F., Ferreras, I., Vazdekis, A., et al. 2013, MNRAS, 433, 3017

La Barbera, F., Vazdekis, A., Ferreras, I., et al. 2017, MNRAS, 464, 3597

La Barbera, F., Vazdekis, A., Ferreras, I., et al. 2019, MNRAS, 489, 4090

Martín-Navarro, I., Vazdekis, A., La Barbera, F., et al. 2015a, ApJ, 806, L31

Martín-Navarro, I., La Barbera, F., Vazdekis, A., Falcón-Barroso, J., \& Ferreras, I. 2015b, MNRAS, 447, 1033

Martín-Navarro, I., La Barbera, F., Vazdekis, A., et al. 2015c, MNRAS, 451, 1081

Martín-Navarro, I., Lyubenova, M., van de Ven, G., et al. 2019, A\&A, 626, A124

McDermid, R. M., Cappellari, M., Alatalo, K., et al. 2014, ApJ, 792, L37

Naab, T., Johansson, P. H., \& Ostriker, J. P. 2009, ApJ, 699, L178

Oser, L., Ostriker, J. P., Naab, T., Johansson, P. H., \& Burkert, A. 2010, ApJ, 725, 2312

Parikh, T., Thomas, D., Maraston, C., et al. 2018, MNRAS, 477, 3954

Pulsoni, C., Gerhard, O., Arnaboldi, M., et al. 2020, A\&A, submitted [arXiv:2009.01823]

Rodriguez-Gomez, V., Pillepich, A., Sales, L. V., et al. 2016, MNRAS, 458, 2371

Sánchez, S. F., Kennicutt, R. C., Gil de Paz, A., et al. 2012, A\&A, 538, A8

Sarzi, M., Spiniello, C., La Barbera, F., Krajnović, D., \& van den Bosch, R. 2018, MNRAS, 478, 4084

Sembach, K. R., \& Tonry, J. L. 1996, AJ, 112, 797

Smith, R. J. 2020, ARA\&A, 58, 577

Spavone, M., Capaccioli, M., Napolitano, N. R., et al. 2017, A\&A, 603, A38

Spiniello, C., Trager, S. C., Koopmans, L. V. E., \& Chen, Y. P. 2012, ApJ, 753, L32

Spiniello, C., Trager, S., Koopmans, L. V. E., \& Conroy, C. 2014, MNRAS, 438, 1483

Spiniello, C., Barnabè, M., Koopmans, L. V. E., \& Trager, S. C. 2015, MNRAS, 452, L21

Spiniello, C., Tortora, C., D’Ago, G., et al. 2021, A\&A, in press, https : //doi . org/10.1051/0004-6361/202038936

Thomas, D., Maraston, C., Bender, R., \& Mendes de Oliveira, C. 2005, ApJ, 621, 673

Treu, T., Auger, M. W., Koopmans, L. V. E., et al. 2010, ApJ, 709, 1195

van Dokkum, P. G., \& Conroy, C. 2010, Nature, 468, 940

van Dokkum, P., Conroy, C., Villaume, A., Brodie, J., \& Romanowsky, A. J. 2017, ApJ, 841, 68

Vaughan, S. P., Davies, R. L., Zieleniewski, S., \& Houghton, R. C. W. 2018, MNRAS, 479, 2443

Vazdekis, A., Casuso, E., Peletier, R. F., \& Beckman, J. E. 1996, ApJS, 106, 307

Vazdekis, A., Koleva, M., Ricciardelli, E., Röck, B., \& Falcón-Barroso, J. 2016, MNRAS, 463, 3409

Veale, M., Ma, C.-P., Thomas, J., et al. 2017, MNRAS, 464, 356 